\title{
Determination of astrophysical nuclear reaction rates using light neutron-rich RNBs
}

\author{
H. Ishiyama ${ }^{\mathrm{a}, 1}$, T. Hashimoto ${ }^{\mathrm{h}}$, T. Ishikawa ${ }^{\mathrm{a}},{\mathrm{K} . \text { Yamaguchi }^{\mathrm{g}} \text {, Y.X. Watanabe }}^{\mathrm{a}}, \mathrm{Y}$.
} Hirayama $^{a}$, N. Imai ${ }^{a}$, H. Miyatake ${ }^{a, b}$, M-H. Tanaka ${ }^{a}$, N. Yoshikawa ${ }^{a}$, S.C. Jeong ${ }^{a}$, Y. Fuchi $^{a}$, T. Nomura ${ }^{a}$, I. Katayama ${ }^{a}$, H. Kawakami ${ }^{a}$, S. Arai ${ }^{a}$, M. Okada ${ }^{a}$, M. Oyaizu ${ }^{\text {, }}$

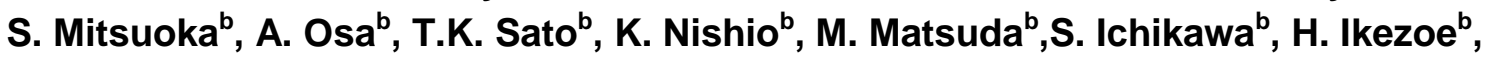

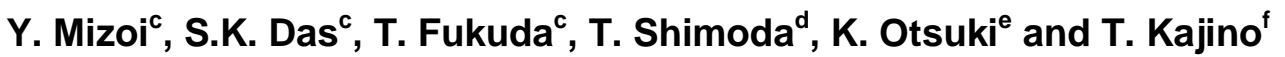

${ }^{a}$ High Energy Accelerator Research Organization (KEK), Tsukuba, Ibaraki, 305-0801 Japan

${ }^{b}$ Japan Atomic Energy Agency (JAEA), Tokai, Ibaraki, 319-1195 Japan

${ }^{c}$ Osaka Electro-Communication University, Neyagawa, Osaka, 572-8530 Japan

${ }^{d}$ Osaka University, Toyonaka, Osaka, 560-0043 Japan

${ }^{e}$ GSI, Planckstrasse 1, 64291 Darmstadt, Germany

${ }^{f}$ National Astronomy Observatory (NAOJ), Mitaka, Tokyo, 181-8588 Japan

${ }^{g}$ University of Tsukuba, Ibaraki, 305-8577 Japan

${ }^{h}$ Tokyo University, Wako, Saitama, 113-0033 Japan

E-mail: hironobu.ishiyama@kek.jp,.hashimoto@cnss.u-tokyo.ac.jp,tomoko@post.kek.jp,

kanako@tac.tsukuba.ac.jp,yutaka.watanabe@kek.jp,yoshikazu.hirayama@kek.jp,nobuaki.imai@kek.jp, hiroari.miyatake@kek.jp,masa-hiko.tanaka@kek.jp,nobuaki.yoshikawa@kek.jp,sunchan.jeong@kek.jp, yoshihide.fuchi@kek.jp,toru.nomura@kek.jp, ichiro.katayama@kek.jp,hirokane.kawakami@kek.jp, shigeaki.arai@kek.jp,masahi.okada@kek.jp,michiharu.oyaizu@kek.jp,mitsuoka.shinichi@jaea.go.jp, osa.akihiko@jaea.go.jp,sato.tetsuya@jaea.go.jp,nishio.katuhisa@jaea.go.jp, matsuda.makoto@jaea.go.jp, ichikawa.shinichi@jaea.go.jp.Ikezoe.hiroshi@jaea.go.jp, mizoi@isc.osakac.ac.jp, suranjan@isc.osakac.ac.jp, fukuda@isc.osakac.ac.jp, tadashi.shimoda@ou.ac.jp, k.otsuki@gsi.de,kajino@nao.ac.jp

Excitation functions of ${ }^{8} \mathrm{Li}(\alpha, \mathrm{n}),(\mathrm{d}, \mathrm{t})$ and ${ }^{12} \mathrm{~B}(\alpha, \mathrm{n})$ reactions were directly measured in the energy region of astrophysical interest using low-energy radioactive nuclear beams of ${ }^{8} \mathrm{Li}$ and ${ }^{12} \mathrm{~B}$. Each measured excitation function is strongly affected by one or more resonances through a compound nucleus. The measured excitation functions are presented. Dominant r-process paths through ${ }^{8} \mathrm{Li}$ at various temperatures are discussed and our future experimental plan is also presented.

10th Symposium on Nuclei in the Cosmos

Mackinac Island, Michigan, USA

27 July-1 August, 2008

\footnotetext{
$1 \quad$ Speaker
} 


\section{Introduction}

It is pointed out that nuclear reactions on light neutron-rich radioactive nuclei play important roles to produce so called 'seed' nuclei and determine the ratio of 'seed' to neutrons at the preceding stage of the r-process. Especially, nuclear reactions through ${ }^{8} \mathrm{Li}$ are thought to be important because of filling a gap of atomic mass number $A=8$ [1]. A systematic study of astrophysical nuclear reaction rates on light neutron-rich nuclei using low-energy radioactive nuclear beams (RNB) is in progress at the tandem facility of Japan Atomic Energy Agency (JAEA). In this report, the measured excitation functions of ${ }^{8} \mathrm{Li}(\alpha, \mathrm{n}),(\mathrm{d}, \mathrm{t})$ and ${ }^{12} \mathrm{~B}(\alpha, \mathrm{n})$ reactions are shown and dominant reaction paths through ${ }^{8} \mathrm{Li}$ during the r-process are discussed. Our future experimental plan is also presented.

\section{Experiment}

There exists two kinds of RNB generators at the tandem facility; one is a recoil mass separator (RMS) as an in-flight secondary beam separator [2]. The other is an ISOL-based RNB facility, named Tokai Radioactive Ion Accelerator Complex (TRIAC) [3], which was constructed and is operated under a joint project of High Energy Accelerator Research Organization (KEK) and JAEA. Using the ${ }^{8} \mathrm{Li}$ and ${ }^{12} \mathrm{~B}$ beams from the RMS with fixed energies of $14.6 \mathrm{MeV}$ and $24 \mathrm{MeV}$, respectively, direct cross-section measurements of ${ }^{8} \mathrm{Li}(\alpha, \mathrm{n}){ }^{11} \mathrm{~B}$ [4] and ${ }^{12} \mathrm{~B}(\alpha, n){ }^{15} \mathrm{~N}$ reactions were performed with a gas chamber surrounded by neutron detector arrays [5]. The gas chamber works not only as a gas counter, but also a He gas target [5]. Using the ${ }^{8} \mathrm{Li}$ beam from the TRIAC with various energies of $0.18-0.75 \mathrm{MeV} / \mathrm{u}$, direct measurement of the ${ }^{8} \mathrm{Li}(\mathrm{d}, \mathrm{t})^{7} \mathrm{Li}$ reaction was carried out using a $\mathrm{CD}_{2}$ target and large-area position-sensitive silicon detectors [6]. For more detailed experimental technique, please see the cited references.

\section{Excitation functions}

The excitation function of the ${ }^{8} \operatorname{Li}(\alpha, n){ }^{11} \mathrm{~B}$ reaction was measured in center-of-mass energies $\left(\mathrm{E}_{\mathrm{cm}}\right)$ from 0.7 to $2.6 \mathrm{MeV}$. The resultant cross sections were roughly two times smaller than previous measurements. A resonance-like structure was found at around $\mathrm{E}_{\mathrm{cm}}=0.85 \mathrm{MeV}$, corresponding to the excited state located at $\mathrm{E}_{\mathrm{x}}=10.9 \mathrm{MeV}$ in ${ }^{12} \mathrm{~B}$. For more detail, please see reference [4].

The excitation function of the ${ }^{12} \mathrm{~B}(\alpha, \mathrm{n}){ }^{15} \mathrm{~N}$ reaction was measured in the energy region of $\mathrm{E}_{\mathrm{cm}}=1.1-3.6 \mathrm{MeV}$, as shown in Fig.1. It covered the Gamow peaks of $\mathrm{T}_{9}=2-5$. The resultant cross sections were almost consistent with the theoretical estimation by Fowler and Hoyle [7]. At $\mathrm{E}_{\mathrm{cm}}=1.4-1.5 \mathrm{MeV}$, a resonance-like structure was observed and may correspond to one or more excited states located at $E_{x}=11.61,11.70,11.75 \mathrm{MeV}$ in ${ }^{16} \mathrm{~N}$. The cross section at $\mathrm{E}_{\mathrm{cm}}=1.5 \mathrm{MeV}$ is about four times larger than the theoretical estimation. The astrophysical reaction rate is directly deduced from measured cross sections by applying the following formula: 


$$
N_{A}<\sigma v>=N_{A}\left(\frac{8}{\pi \mu}\right)^{1 / 2} \frac{1}{k T^{3 / 2}} \int_{0}^{\infty} \sigma(E) E \exp \left(-\frac{E}{k T}\right) d E
$$

Where $\sigma(E)$ is the cross section, $N_{A}$ is Avogadro's number, $m$ is the reduced mass, $k$ is Boltzmann's constant, and $\mathrm{T}$ is the temperature. In the energy region below $\mathrm{E}_{\mathrm{cm}}=1.1 \mathrm{MeV}$ and above $3.8 \mathrm{MeV}$, we used cross section data estimated by Fowler and Hoyle. The resultant reaction rate is roughly two times faster at around $\mathrm{T}_{9}=3$ than the theoretical estimation [7].

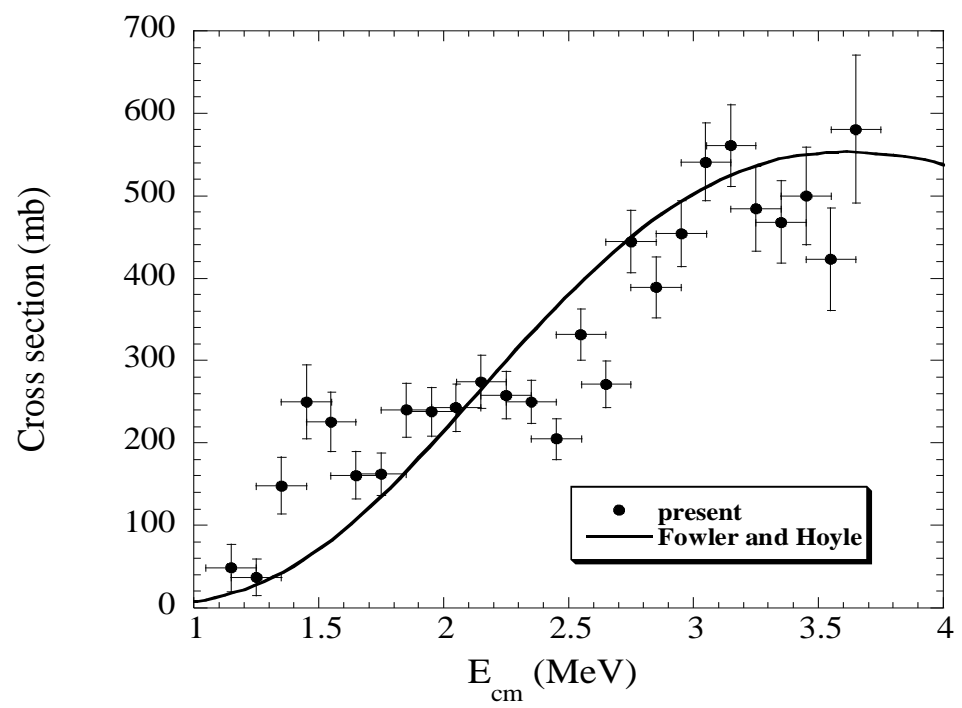

Figure 1: Excitation function of the ${ }^{12} \mathrm{~B}(\alpha, n){ }^{15} \mathrm{~N}$ reaction. Black circles show present results. The solid line indicates the theoretical estimation by Fowler and Hoyle [7].

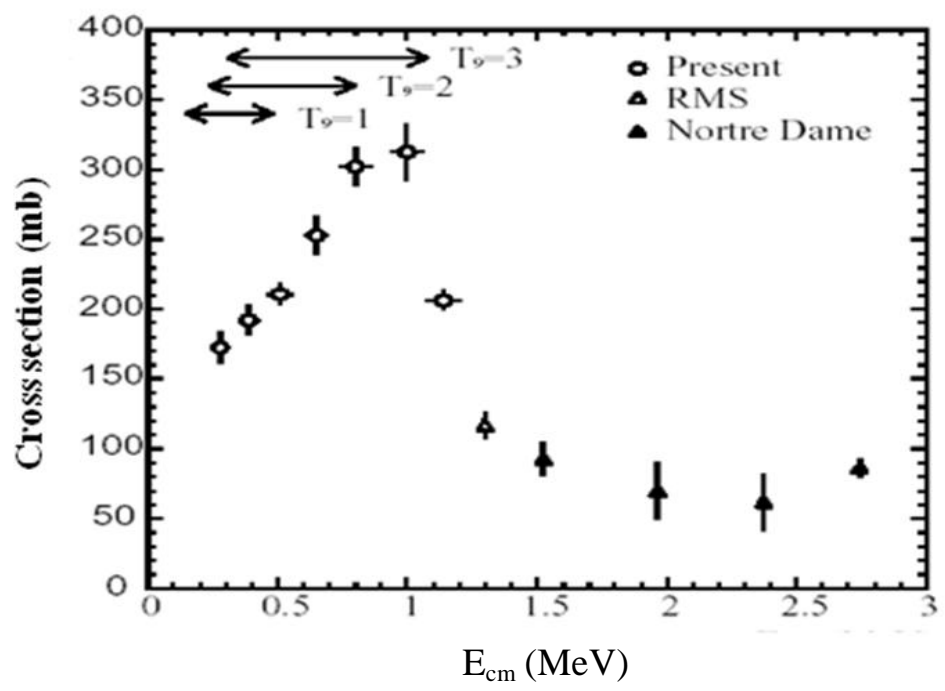

Figure 2: Excitation function of the ${ }^{8} \mathrm{Li}(\mathrm{d}, \mathrm{t})^{7} \mathrm{Li}$ reaction. Open circles show the present results. The open triangle shows our measurement using the ${ }^{8} \mathrm{Li}$ beam from the RMS. Black triangles indicate the previous results by Balbes et al. [8]. 
The excitation function of the ${ }^{8} \mathrm{Li}(\mathrm{d}, \mathrm{t})^{7} \mathrm{Li}$ reaction was measured in the energy region of $\mathrm{E}_{\mathrm{cm}}=0.3-1.2 \mathrm{MeV}$, as shown in Fig. 2. It covers the Gamow peaks of $\mathrm{T}_{9}=1-3$. Previous measurement by Balbes et al. [8] was performed in higher energy region over $\mathrm{E}_{\mathrm{cm}}=1.5 \mathrm{MeV}$. At around $\mathrm{E}_{\mathrm{cm}}=0.8 \mathrm{MeV}$, a resonance-like structure was observed and its energy corresponds to the $\mathrm{E}_{\mathrm{x}}=22.4 \mathrm{MeV}$ state in ${ }^{10} \mathrm{Be}$. The reaction rate was deduced from present data by applying the formula (1). In the energy region above $1.5 \mathrm{MeV}$, we used the cross section data in previous measurement [8]. The cross section below $\mathrm{E}_{\mathrm{cm}}=0.3 \mathrm{MeV}$ were estimated by linear extrapolation from the present data point at $\mathrm{E}_{\mathrm{cm}}=0.3 \mathrm{MeV}$ to $0.0 \mathrm{MeV}$. The resultant rate is higher by one order of magnitude at around $\mathrm{T}_{9}=1$ than the previously reported values [8] due to the resonance-like structure around $\mathrm{E}_{\mathrm{cm}}=0.8 \mathrm{MeV}$.

\section{Reaction rates and dominant reaction paths via ${ }^{8} \mathrm{Li}$}

In order to identify main flow paths through ${ }^{8} \mathrm{Li}$ at various temperatures during the $\mathrm{r}$ process, relative reaction rates $\left(\mathrm{Y}_{\mathrm{X}} \mathrm{Y}_{8 \mathrm{Li}}<\sigma \mathrm{v}>\right)$ on ${ }^{8} \mathrm{Li}$ were calculated, as shown in Fig. 3. The $\mathrm{Y}_{\mathrm{x}}$ is fraction of each light element, proton $\left(\mathrm{Y}_{\mathrm{p}}\right)$, neutron $\left(\mathrm{Y}_{\mathrm{n}}\right)$, deuteron $\left(\mathrm{Y}_{\mathrm{d}}\right)$ and alpha particle $\left(\mathrm{Y}_{\alpha}\right)$. Those values were deduced by a network calculation in the r-process using the exponential model [9]. Initial parameters of the network calculation were set at Ye (electron fraction) $=0.45$, $\tau_{\text {dye }}($ dynamic time scale $)=5 \mathrm{~ms}$ and $\mathrm{s} / \mathrm{k}($ entropy $)=250$. Those values are typical ones to reproduce the r-process abundances under the neutrino-driven wind model in the Type II supernovae. The $\mathrm{Y}_{8 \mathrm{Li}}$ is fraction of ${ }^{8} \mathrm{Li}$ and is set to unity. The reaction rates of ${ }^{8} \mathrm{Li}(\mathrm{d}, \mathrm{t})$ and ${ }^{8} \operatorname{Li}(\alpha, n)$ are deduced from present results. $\operatorname{The}{ }^{8} \operatorname{Li}(\mathrm{p}, \alpha)$ and the ${ }^{8} \operatorname{Li}(\mathrm{n}, \gamma)$ rates are from references [10] and [11], respectively.

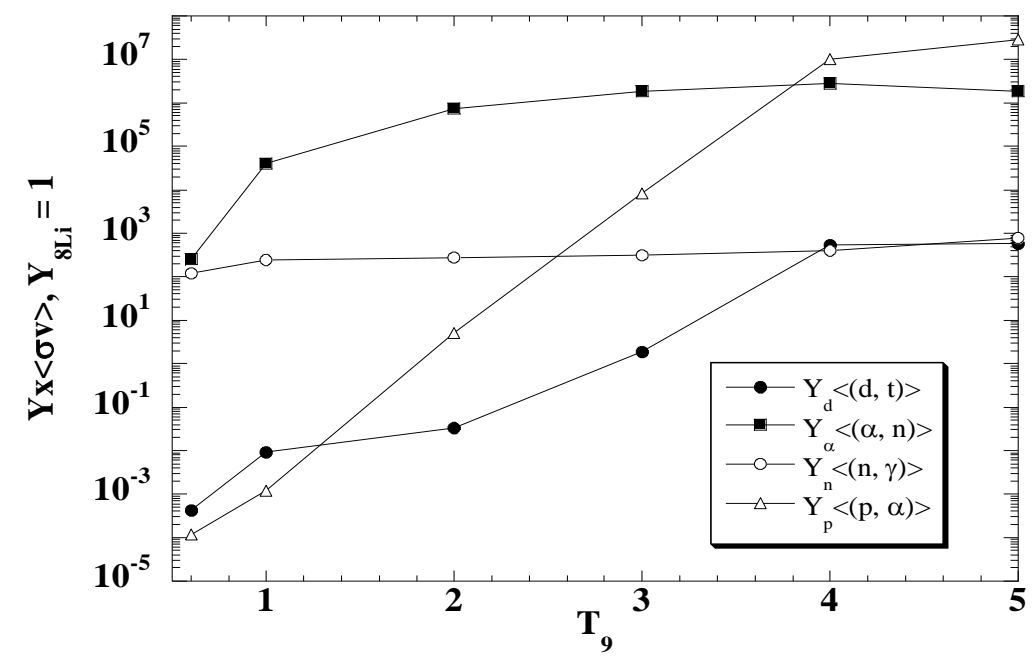

Figure 3: Relative reaction rates $\left.\left(\mathrm{Y}_{\mathrm{x}} \mathrm{Y}_{8 \mathrm{Li}}<\sigma \mathrm{v}\right\rangle\right)$ on ${ }^{8} \mathrm{Li}$. The $\mathrm{Y}_{\mathrm{x}}$ is fraction of light element and the $\mathrm{Y}_{8 \mathrm{Li}}$ is fraction of ${ }^{8} \mathrm{Li}$. For more detail, please see the text. 
As can be seen in Fig. 3, at $\mathrm{T}_{9}>3.7$, the ${ }^{8} \mathrm{Li}(\mathrm{p}, \alpha) \alpha$ reaction is the fastest reaction, which destroys the ${ }^{8} \mathrm{Li}$. In $\mathrm{T}_{9}=0.7-3.7$, the ${ }^{8} \mathrm{Li}(\alpha, \mathrm{n})^{11} \mathrm{~B}$ reaction becomes the main path from the ${ }^{8} \mathrm{Li}$. The ${ }^{8} \mathrm{Li}(\mathrm{d}, \mathrm{t})^{7} \mathrm{Li}$ rate is so slow that this reaction gives little effect to the r-process abundances.

The relative reaction rates on ${ }^{11} \mathrm{~B}$ and ${ }^{12} \mathrm{~B}$ were calculated with the above mentioned procedure. As the result, dominant reaction paths through ${ }^{8} \mathrm{Li}$ at various temperatures are identified as below;

$$
\begin{aligned}
& \mathrm{T}_{9}=2.7-3.6:{ }^{8} \operatorname{Li}(\alpha, \mathrm{n}){ }^{11} \mathrm{~B}(\mathrm{p}, \alpha){ }^{8} \mathrm{Be}(2 \alpha), \\
& \mathrm{T}_{9}=1.7-2.7:{ }^{8} \operatorname{Li}(\alpha, \mathrm{n}){ }^{11} \mathrm{~B}(\alpha, \mathrm{n})^{14} \mathrm{~N}, \\
& \mathrm{~T}_{9}=0.5-1.7:{ }^{8} \operatorname{Li}(\alpha, \mathrm{n}){ }^{11} \mathrm{~B}(\mathrm{n}, \gamma){ }^{12} \mathrm{~B}(\mathrm{n}, \gamma){ }^{13} \mathrm{~B} .
\end{aligned}
$$

\section{Future plan}

The measured cross sections of the ${ }^{8} \mathrm{Li}(\alpha, \mathrm{n}){ }^{11} \mathrm{~B}$ reaction have relatively large errors of 20-30\% [4] in the energy region below $\mathrm{E}_{\mathrm{cm}}=1.0 \mathrm{MeV}$, corresponding to $\mathrm{T}_{9}=1-2$. To improve statistics and energy resolution of cross sections, we have a plan to measure the cross sections below $\mathrm{E}_{\mathrm{cm}}=1.0 \mathrm{MeV}$ using the ${ }^{8} \mathrm{Li}$ beam from the TRIAC with the intensity of $10^{5-6} \mathrm{pps}$ and the energy resolution of $2 \%$. The present gas chamber, named MSTPC [5], works well up to $10^{4}$ pps injection-rate. Under higher injection rate, the gain instability occurs due to space charge gain limitation around anode wires. We therefore decided to exchange the anode wires for gaselectron-multiplier (GEM) foils for high-rate capability. For experimental requirement, gas multiplication of the GEM-MSTPC should be enough high (over $10^{3}$ ) with $\mathrm{He}+\mathrm{CO}_{2}(10 \%)$ gas and low gas pressure (about 100 Torr). A $400 \mu \mathrm{m}$ thick GEM foil was selected and gave $10^{3}$ gas gain successfully. An off-line test of the GEM-MSTPC for higher rate capability is in progress.

\section{References}

[1] T. Sasaqui, et al., Astrophys. J. 634(2005)1173.

[2] H. Ishiyama, et al., Nucl. Instrum. Methods A560(2006)366.

[3] H. Miyatake, et al., Nucl. Instrum. Methods B204(2003)746.

[4] H. Ishiyama, et al., Phys. Lett. B640(2006)82.

[5] T. Hashimoto, et al., Nucl. Instrum. Methods A556(2006)366.

[6] T. Hashimoto, et al., AIP Conf. Proc. 1016(2008)313.

[7] W.A. Fowler and F. Hoyle, Astrophys. J. Suppl. 91(1964)201.

[8] M.J. Balbes, et al., Nucl. Phys. A584(1995)315.

[9] K. Otsuku, et al., New Astronomy 8(2003)767

[10] F.D. Becchetti, et al., Nucl. Phys. A550(1992)507.

[11] Z.H. Liu, et al., Phys. Rev. C71(2005)052801. 